\section{ANALYSIS AND DESIGN OF A WIDE BAND OMNI- DIRECTIONAL ANTENNA}

\section{O.B. Jacobs, J.W. Odendaal and J. Joubert}

Centre for Electromagnetism, Department of Electrical, Electronic and Computer Engineering, University of Pretoria, Pretoria 0002,

Corresponding author: s26249822@tuks.co.za

\begin{abstract}
The analysis and design of an omni-directional antenna that functions over a very broad frequency band is presented. Design guidelines for this antenna have been derived using electromagnetic analysis software. Measured results of a prototype developed according to these guidelines are presented. These results are compared to simulation results to illustrate the validity of the model used to derive the empirical design guidelines.
\end{abstract}

Key Words: asymptotic conical dipole; omni-directional antenna; wideband antenna.

\section{INTRODUCTION}

Omni-directional antennas that are capable of functioning over a very broad band are required in many applications, especially in wireless telecommunications systems [1]. Other applications of interest include electronic countermeasures and as calibration antennas for electromagnetic compatibility testing [2].

A simple structure that is often used to obtain a broad band omnidirectional antenna is the biconical antenna. The input impedance of an infinite biconical antenna is constant and depends only on the angle of the cones. For finite cones, however, the impedance has an oscillatory variation as a function of frequency [3]. In addition the impedance of typical biconical antennas is much higher than the $50 \Omega$ impedance of a typical coaxial line which necessitates the use of impedance transformers [4]. Obtaining a biconical antenna with lower impedance is possible but only at a cost of making the antenna structure impractical as the cone half angle required to achieve an impedance of $50 \Omega$ results in a very large and unwieldy structure [5].

The Schelkunoff-Friis antenna also known as the asymptotic conical dipole (ACD) antenna is a geometry that was first proposed in 1952 [6]. The antenna surface is defined as an equipotential contour due to a line of charge and is a tapered structure. By changing the charge density of the line and the voltage for which the contour is defined, different contours can be obtained $[7,8]$. The contour is then spun around its axis to obtain a three dimensional shape, and Figure 1 shows examples of a biconical and an ACD antenna.
These antennas have been extensively used for low frequency electrically small probes [9] and for generating electromagnetic pulses [8]. Design and analysis of the antenna for this use has been done using a quasi-static analysis approach [7]. Design descriptions of the antenna for use at higher frequencies as a radiating structure have been lacking or have been based on unjustified assumptions.
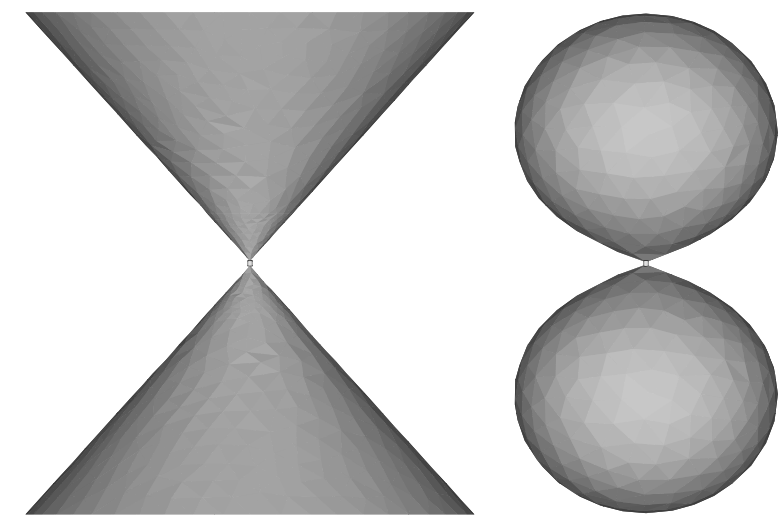

Figure 1 A biconical antenna (left), and an asymptotic conical dipole antenna (right).

In this article commercially available software (FEKO [10]) is used to accurately model the antenna using full wave analysis. This model was used to derive a basic set of design guidelines for an antenna with a realistic feed structure. A truncated version of the antenna is also presented that aims at making the antenna smaller and easier to manufacture. A prototype truncated ACD antenna was designed according to these guidelines. Measurements of the prototype antenna are also presented.

\section{MODEL OF THE ANTENNA}

The ACD is a surface that is obtained by calculating an equipotential contour for a fixed line of charge. The contour can be determined using the following Eqs. [6 8].

$$
\begin{gathered}
r=h\left[\frac{\left(1+e^{\frac{4 \pi \varepsilon_{0} V}{q}}\right) \cos \theta+\left(1-e^{\frac{4 \pi \varepsilon_{0} V}{q}}\right)}{\left(1+e^{\frac{4 \pi \varepsilon_{0} V}{q}}\right) \cos \theta+\left(1-e^{\frac{4 \pi \varepsilon_{0} V}{q}}\right) \cos ^{2} \theta}\right] \\
\theta_{\max }=\cos ^{-1}\left(\frac{e^{\frac{4 \pi \varepsilon_{0} V}{q}}-1}{1+e^{\frac{4 \pi \varepsilon_{0} V}{q}}}\right)
\end{gathered}
$$


The height of the line $(h)$, the charge of the line $(q)$ and the potential $(V)$ of the contour are the parameters that determines the shape of the contour (in polar coordinates). $\theta_{\max }$ is the angle up to which Eq. (1) is evaluated to determine the contour. By spinning the contour around a central axis a three dimensional structure can be obtained.

The antennas were analyzed using the computational electromagnetic code FEKO [10]. Two models were used to simulate the antenna. A simplified model using a delta source feed was used to derive the design guidelines, whereas a model that included detail of the coaxial feed section of the antenna was used for the final antenna design. The feed section of this model is shown in Figure 2. The detailed feed model is a representation of the antenna fed using a coaxial cable. The inner is connected to the top portion of the antenna and the outer to the bottom. The dielectric of the coaxial cable was used as a spacer between the two parts of the antenna and to provide some additional structural support.

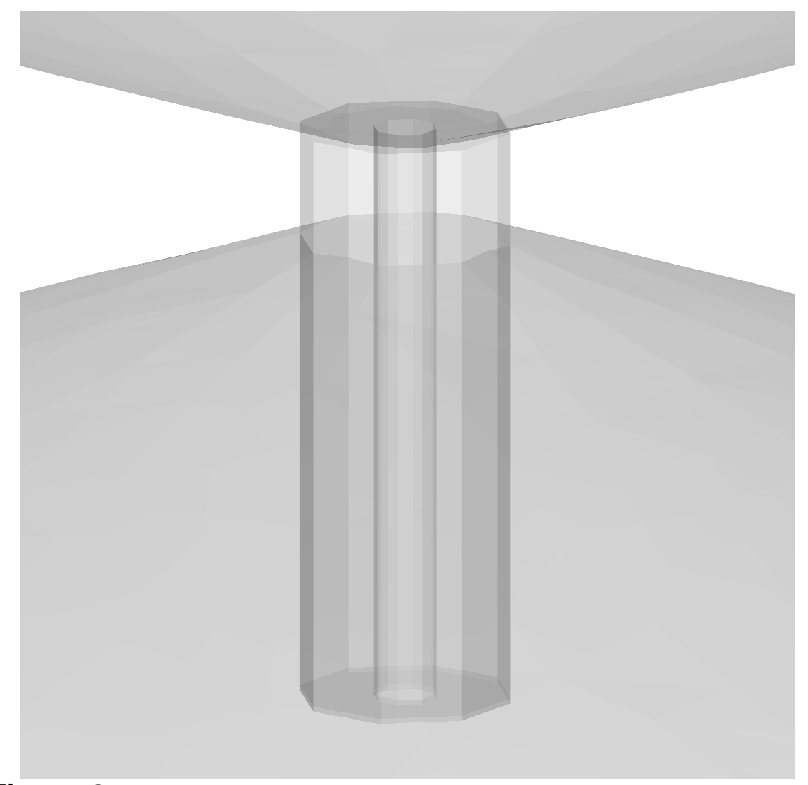

Figure 2 A detailed view of the feed used for the ACD antenna.

\section{ANTENNA DESIGN GUIDELINES}

Design of an ACD antenna requires the determination of the appropriate height of the antenna as a fraction of the lowest wavelength of operation as well as the shape of the antenna to obtain specific input impedance.

\subsection{Antenna Impedance}

Using quasi static analysis the characteristic impedance of an ACD antenna can be approximated as in Eq. (3) [7]. Where $n$ is a constant, $q$ is the charge of the line and $V$ is the potential.

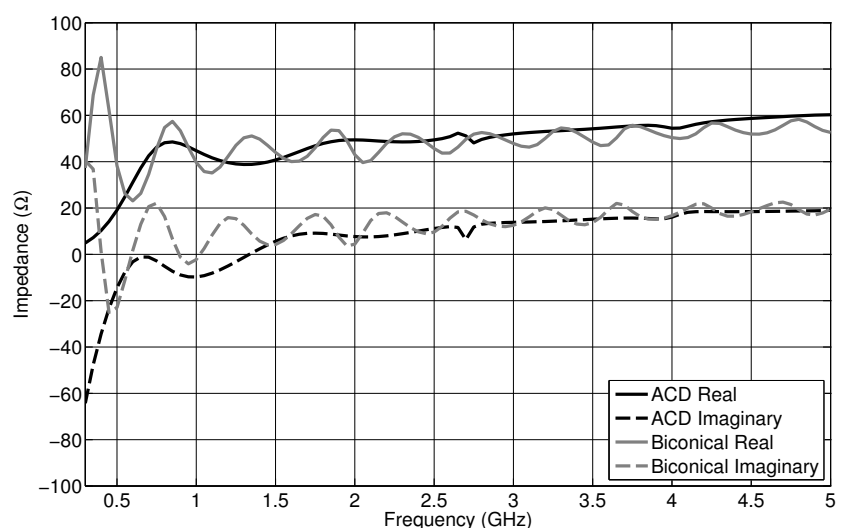

Figure 3 Comparison of biconical antenna real and imaginary impedance with ACD antenna real and imaginary impedance.

$$
Z=\frac{n V}{c_{0} q}
$$

Using $n=1$ the impedance of a monopole above a ground plane can be approximated while $n=2$ for a dipole. A parametric study was conducted to determine whether this relation would be an adequate design guideline for the input impedance of an ACD antenna. The effect of changing parameters $V$ and $q$ on the input impedance was investigated using the FEKO model of the antenna. This indicated that although the relation above gives an approximation to the input impedance a given ACD antenna with parameters $V$ and $q$ the approximation of the impedance with a physically realizable feed structure can be greatly improved by changing $n$ to 2.5 for a dipole and 1.25 for a monopole.

\subsection{Antenna Height}

Simulations with antennas of varying heights indicated that a suitable height of the antenna to obtain a standing wave ration better than 2:1 should be approximately a third of the wavelength of the lowest frequency of operation as opposed to a previous estimate of a quarter of a wavelength [7]. It should be noted that for the design of ACD antennas that are electrically large the antenna radiation pattern can contain broadside nulls even if the antenna is matched to the source and such antennas would have to be analyzed to identify the frequencies at which such nulls occur.

To be able to feed the antenna with a coaxial cable as shown in Figure 2 and to reduce the weight of the structure the effect of truncating the antenna was also investigated. It was found that cutting away up to 15 
percent of the height of the antenna only slightly reduced the impedance bandwidth of the antenna and had very little effect on the radiation patterns.

\subsection{Comparison with a biconical antenna}

The simulation results of the antenna indicate the advantages of this structure over a biconical antenna. The input impedance of a biconical antenna designed to be matched to a $50 \Omega$ source is compared to that of a truncated ACD antenna in figure 3. This indicates that the ACD antenna has smaller impedance variations as a function of frequency.

The greatest improvement is, however, the size of the antenna. The models of the biconical antenna and the

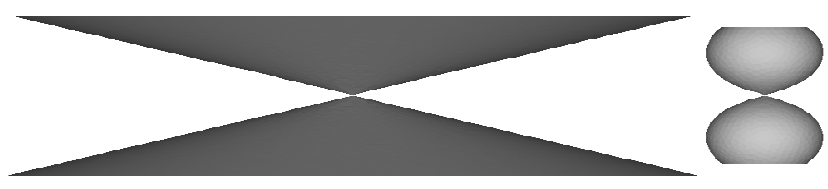

Figure 4 Size comparison of the biconical (left) and truncated ACD antenna model (right) for an input impedance of $50 \Omega$.

truncated ACD antenna that were simulated to obtain the results in Figure 3 are shown for comparison in Figure 4.The width of the truncated ACD antenna is less than a fifth of the biconical antenna with the same impedance.

\section{ANTENNA PROTOTYPE DESIGN AND MEASUREMENTS}

The design guidelines for the ACD antenna were verified by developing a prototype. The antenna was designed to operate from a frequency of $300 \mathrm{MHz}$. A third of a wavelength at this frequency is $333 \mathrm{~mm}$. The total height of the antenna was taken slightly larger than this at $340 \mathrm{~mm}$ to account for the slight degradation in performance due to the truncation of the structure. The structure was then truncated down by $12 \%$ to a height of $300 \mathrm{~mm}$.

The required input impedance of $50 \Omega$ was achieved by using parameters $\mathrm{V}=80$ and $\mathrm{q}=13.33 \times 10^{-9}$. The diameter of the resulting structure was $170 \mathrm{~mm}$. The antenna body was machined from aluminum and used a coaxial SMA connector as input to the feed section. The side wall thickness of the antenna was $5 \mathrm{~mm}$.

The simulated and measured VSWR is shown in figure 5 and are in good agreement over the frequency range $300 \mathrm{MHz}$ to $18 \mathrm{GHz}$ with a low standing wave ratio over this bandwidth.

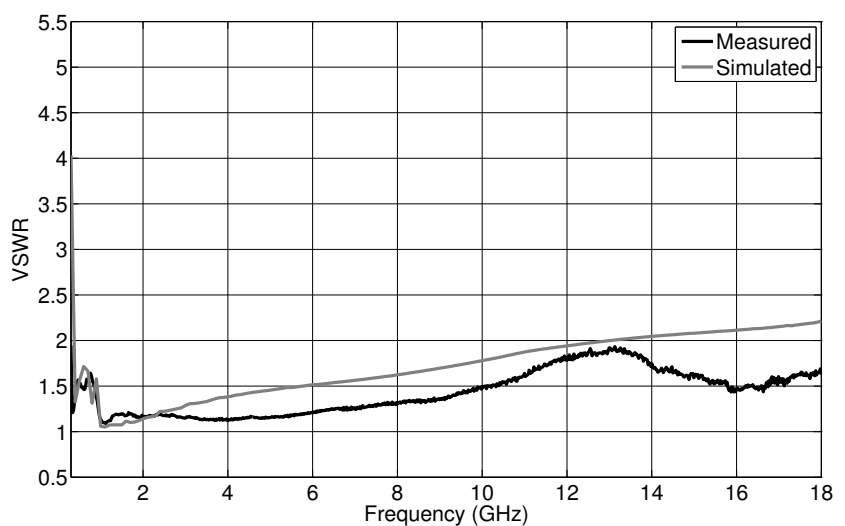

Figure 5 Measured and simulated VSWR of the truncated ACD antenna designed for the frequency range $300 \mathrm{MHz}$ to $18 \mathrm{GHz}$.

The measurement was conducted inside an anechoic chamber using an HP8510 Vector Network Analyzer.

The measured antenna radiation patterns also show good agreement with simulated results as shown in Figure 6.

The radiation patterns were measured in a compact antenna test range from $1 \mathrm{GHz}$ to $18 \mathrm{GHz}$. The radiation patterns have a beamwidth that varies little with frequency and maintains good omnidirectional patterns. At higher frequencies diffraction effects or ripple in the radiation pattern is observed due to the sharp edge of the truncated structure.

A comparison between the measured and simulated bore sight gain is shown in Figure 7 and reflects good agreement between measured and simulated results. The nominal bore sight gain is $3 \mathrm{dBi}$.

\section{CONCLUSION}

Computational electromagnetic software was used to analyze the ACD antenna. A refined impedance design guideline for this antenna was presented. It was found that the design parameter $n$ should be adjusted to 1.25 for a monopole above a ground plane and to 2.5 for a dipole structure. A design guideline for the height of the antenna was derived from the results of a parametric study. A truncated version of this antenna up to $15 \%$ smaller than the full ACD antenna was proposed. This made the antenna smaller and easier to manufacture. The design guidelines were then used to design a prototype antenna that verified the antenna models used as well as the design procedure.

The prototype indicated the extremely broad band operation of the antenna and highlighted the advantages this structure offers over typical biconical antennas, namely more stable impedance as a function of frequency and the ability to design a practical antenna 


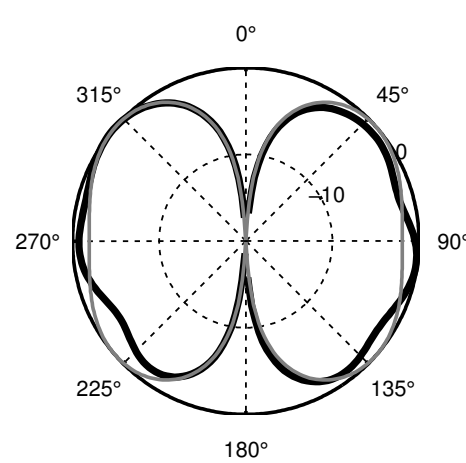

(a)

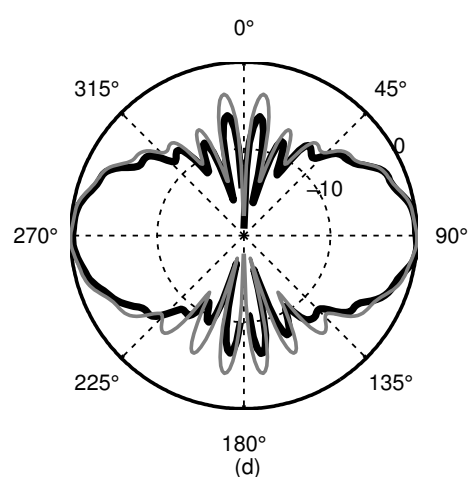

$0^{\circ}$

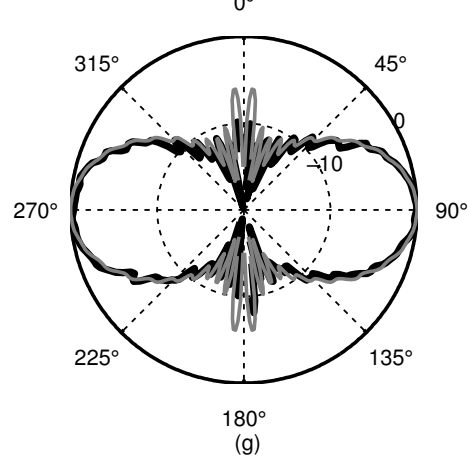

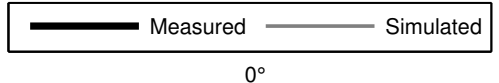
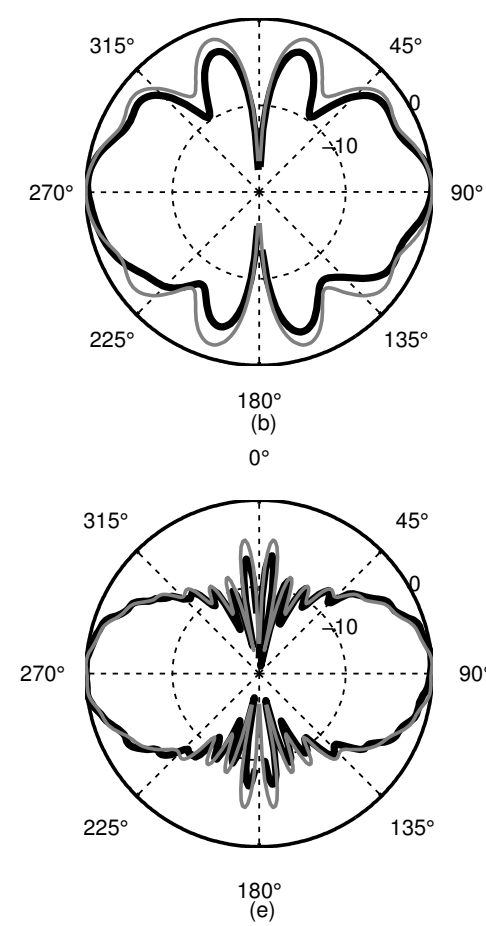

(e)

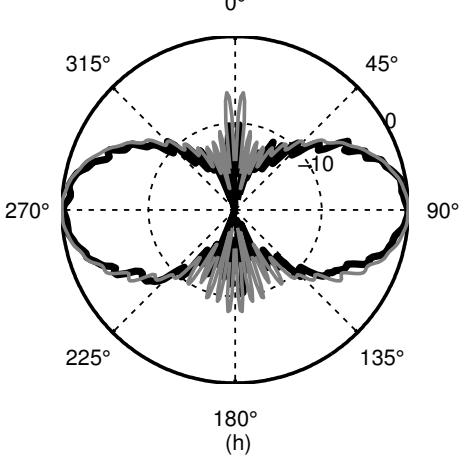

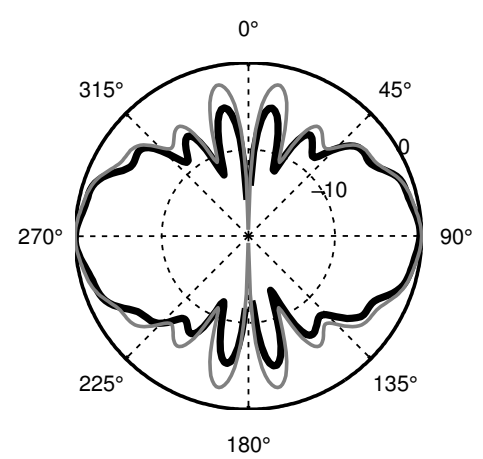

(c)

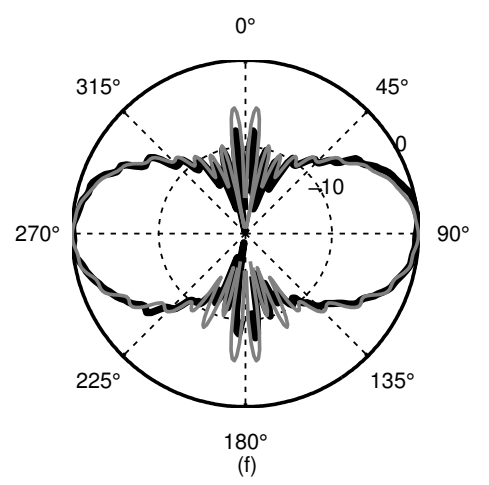

$0^{\circ}$

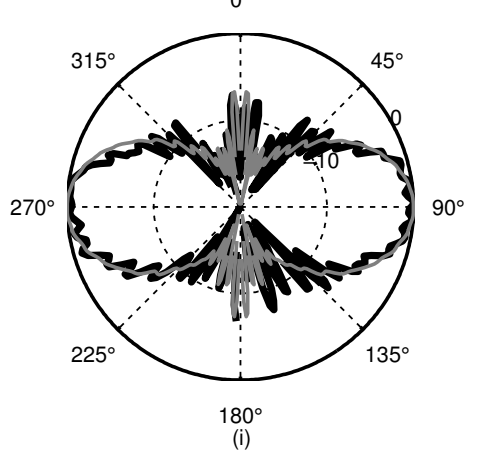

Figure 6 Radiation patterns of the truncated ACD antenna at (a) $1 \mathrm{GHz}$, (b) $3 \mathrm{GHz}$, (c) $5 \mathrm{GHz}$, (d) $7 \mathrm{GHz}$, (e) $9 \mathrm{GHz}$, (f) $11 \mathrm{GHz}$, (g) $13 \mathrm{GH}$, (h) $15 \mathrm{GHz}$ and (i) $17 \mathrm{GHz}$.

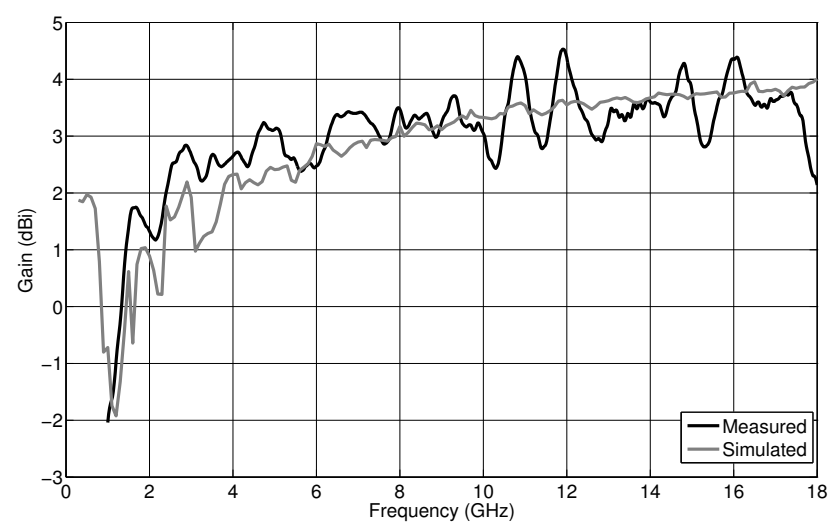

Figure 7 Measured and simulated bore sight gain of the truncated ACD antenna. that has small physical dimensions, especially a small width, and a low weight while still being well matched to a $50 \Omega$ source. The antenna has a nominal gain of 3 $\mathrm{dBi}$ and a measured VSWR less than 2:1 over the frequency range $300 \mathrm{MHz}$ to $18 \mathrm{GHz}$.

\section{ACKNOWLEDGEMENTS}

The authors thank EMSS-SA who provided valuable support and insight on the use of FEKO. 


\section{REFERENCES}

1. J. Ma, Y. Z. Yin, S. G. Zhou and L. Y. Zhao, Design of a new wideband low-profile conical antenna, Microwave and Opt Technol Lett 51 (2009), 2620-2623.

2. A. R. Mallahzadeh, R. Pazoki and S. Karimkashi, A new UWB skeletal antenna for EMC applications, Appl Comput Electrom 23 (2008), 352-356.

3. C.A. Balanis, Antenna Theory: Analysis and Design, 3rd ed., John Wiley \& Sons, Hoboken, NJ, 2005.

4. D.N. Black and T.A. Brunasso., An Ultra-Wideband Bicone Antenna, IEEE Int Conf Ultra-Wideband - Proc (2006), pp. 327-332.

5. A. Kazemipour, S. Begaud and S.H. Kim, Feasibility of a wideband calculable standard antenna for EMC measurements, Conf Precision Electromagn Measurements Dig (2008), pp. 434-435.
6. S.A. Schelkunoff. and H.T. Friis, Antennas, Theory and Practice, John Wiley \& Sons, New York, 1952.

7. T.L. Simpson, Analysis and design of the Schelkunoff-Friis antenna, IEEE Antennas Propagat Soc Int Symp (2006), pp 611-614.

8. N.C. De, T.K. Ghosh, D.R. Poddar, and S.K. Chowdhury, Design and Experimental Investigation of the Asymptotic Conical dipole Antenna, IEEE Trans Electromagn Compat, 37 (1995), pp. 282-285.

9. B. Kordi, J. Lo Vetri, G. Bridges, and L. Jeffrey, Calibration methods for electric field probes and GTEM cells, Int Symp Electromagn Compat 3 (2004), pp. 908-912.

10. EM Software \& Systems, FEKO User's Manual, 2008. 